\title{
The Signless Laplacian Spectral Radius for Bicyclic Graphs with $k$ Pendant Vertices
}

\author{
LIHUA FENG
}

School of Mathematics, Shandong Institute of Business and Technology, 191 Binhaizhong Road, Yantai, Shandong 264005, P. R. China

e-mail : fenglh@163.com

ABSTRACT. In this paper, we study the signless Laplacian spectral radius of bicyclic graphs with given number of pendant vertices and characterize the extremal graphs.

\section{Introduction}

Let $G=(V, E)$ be a simple connected graph with vertex set $V=\left\{v_{1}, v_{2}, \cdots, v_{n}\right\}$ and edge set $E$. The order of a graph is the cardinality of its vertex set. The matrix $Q(G)=D(G)+A(G)$ is called the signless Laplacian matrix of graph $G$, where $D(G)=\operatorname{diag}\left(d_{u}, u \in V\right)$ is the diagonal matrix of vertex degrees of $G$ and $A(G)$ is the adjacency matrix of $G$. It is known that $Q(G)$ is a positive semi-definite matrix, we call this matrix the $Q$-matrix and its largest eigenvalue is denoted by $\mu(G)$ or $\mu$ for simplicity. For the background on the Laplacian eigenvalues of a graph, the reader is referred to [20] and the references therein.

It is well known that the matrix $L(G)=D(G)-A(G)$ is called the Laplacian matrix, and $\lambda(G) \leq \mu(G)$ (see, for example, [14]), the equality holds if and only if $G$ is bipartite.

A bicyclic graph is a connected graph with vertex number equal to edge number minus one. A pendant path in a connected graph is a path attached to a connected graph. For $S \subset V, G[S]$ denotes the subgraph induced by $S$. For $u \in V, d_{u}$ is the degree of $u, N(u)$ is the neighbor set of $u$.

Denote by $C_{n}$ and $P_{n}$ the cycle and the path on $n$ vertices, respectively. We will use $\mathcal{B}_{n}(k)$ to denote the set of bicyclic graphs on $n$ vertices with $k$ pendant vertices. Let $C_{p}$ and $C_{q}$ be two vertex disjoint cycles. Suppose that $v_{1}$ is a vertex of $C_{p}$ and $v_{t}$ is a vertex of $C_{q}$. Joining $v_{1}$ and $v_{t}$ by a path $v_{1} v_{2} \cdots v_{t}$ of length $t-1$, where $t \geq 1$ and $t=1$ means identifying $v_{1}$ with $v_{t}$, the resulting graph, denoted by $B(p, t, q)$. The set of bicyclic graphs obtained from $B(p, t, q)$ by attaching trees is denoted by $\mathcal{B}_{n}^{+}(k)$. Let $P_{t+1}, P_{p+1}$ and $P_{q+1}$ be three vertex-disjoint paths, where $t, p, q \geq 1$ and at most one of them is 1 . Identifying the three initial vertices and

Received August 3, 2009; revised September 30, 2009; accepted October 9, 2009.

2000 Mathematics Subject Classification: 05C50, 15A18.

Key words and phrases: Bicyclic graph; signless Laplacian; spectral radius; pendant vertex. This paper was supported Foundation of Education Committee of Shandong Province (J07YH03) and NSFSD(No. Y2008A04). 
terminal vertices of them, respectively, the resulting graph is denoted by $P(t, p, q)$. The set of bicyclic graphs obtained from $P(t, p, q)$ by attaching trees is denoted by $\mathcal{B}_{n}^{++}(k)$. Obviously, $\mathcal{B}_{n}(k)=\mathcal{B}_{n}^{+}(k) \cup \mathcal{B}_{n}^{++}(k)$. For other notations in graph theory, we follow [1].

The Laplacian spectral radius of unicyclic graphs is well studied. In [17], the upper and lower bounds for Laplacian spectral radius of unicyclic graphs were studied. In [13], the author characterized the maximum Laplacian spectral radius of unicyclic graphs with fixed girth. In [18], the Laplacian spectral radius of bicyclic graphs were studied. In [16], the spectral radius of bicyclic graphs with given number of pendant vertices were studied. The study of the signless Laplacian spectral radius attracts researchers attention just recently. In [7], Fan et.al. studied the signless Laplacian spectral radius of bicyclic graph with fixed order. In [6], the authors discussed the smallest eigenvalue of $Q(G)$ as a parameter reflecting the nonbipartiteness of the graph $G$. Some other use of the signless Laplacian can be found in [12], [3]. For a survey paper of this new direction, see [5].

In this paper, we study the Laplacian spectral radius of bicyclic graphs with given pendant vertices. We also characterize the extremal graphs.

\section{Some lemmas}

Lemma 2.1([19]). Let $G$ be a connected graph and $u, v$ be two vertices of $G$. Suppose $v_{1}, v_{2}, \cdots, v_{s} \in N(v) \backslash(N(u) \cup\{u\})\left(1 \leq s \leq d_{v}\right)$, and $G^{*}$ is the graph obtained from $G$ by deleting the edges vv $v_{i}$ and adding the edges $u v_{i}(1 \leq i \leq s)$. Let $X=\left(x_{1}, x_{2}, \cdots, x_{n}\right)^{t}$ be the principal eigenvector of $Q(G)$, where $x_{i}$ corresponds to $v_{i}(1 \leq i \leq n)$. If $x_{u} \geq x_{v}$, then $\mu(G)<\mu\left(G^{*}\right)$.

We generalize Lemma 2.1 next.

Lemma 2.2. Let $G$ be a connected graph of order $n$ and $S, T$ be its two disjoint nonempty vertex subset. Suppose $S=\left\{v_{1}, v_{2}, \cdots, v_{s}\right\}$ and the neighbors of $v_{i}$ in $T$ are $v_{i 1}, v_{i 2}, \cdots, v_{i l_{i}}\left(l_{i} \geq 1, i=1,2, \cdots, s\right)$. Let $X=\left(x_{v_{1}}, x_{v_{2}}, \cdots, x_{v_{n}}\right)^{t}$ be the Perron vector of $Q(G)$, where $x_{v_{k}}$ corresponds to the vertex $v_{k}(1 \leq k \leq n)$. Suppose

$x_{v_{1}}=\max \left\{x_{v_{i}}: i=1,2, \cdots, s\right\}$. Let $H$ be the graph obtained from $G$ by deleting edges $v_{i} v_{i j}$ and adding the edges $v_{1} v_{i j}\left(i=2,3, \cdots, s ; j=1,2, \cdots, l_{i}\right)$. Then we have $\mu(G)<\mu(H)$.

Proof. The proof is similar to that in [11], we present it here for completeness. Obviously,

$$
\begin{aligned}
X^{t}(Q(H)-Q(G)) X & =X^{t}(D(H)+A(H)-D(G)-A(G)) X \\
& =\sum_{i=2}^{s} \sum_{j=1}^{l_{i}}\left(\left(x_{v_{1}}+x_{v_{i j}}\right)^{2}-\left(x_{v_{i}}+x_{v_{i j}}\right)^{2}\right)
\end{aligned}
$$




$$
\begin{aligned}
& =\sum_{i=2}^{s} \sum_{j=1}^{l_{i}}\left(\left(x_{v_{1}}^{2}-x_{v_{i}}^{2}\right)+2 x_{v_{i j}}\left(x_{v_{1}}-x_{v_{i}}\right)\right) \\
& \geq 0
\end{aligned}
$$

Thus,

$$
\mu(H)=\max _{\|Y\|=1} Y^{t} Q(H) Y \geq X^{t} Q(H) X \geq X^{t} Q(G) X=\mu(G) .
$$

If $\mu(H)=\mu(G)$, then the inequalities above should be equalities. So

$$
\mu(H)=X^{t} Q(H) X=X^{t} Q(G) X=\mu(G) .
$$

Thus, $\mu(H) X=Q(H) X$ and $Q(G) X=\mu(G) X$. Thus,

$$
\begin{aligned}
& \mu(H) x_{v_{1}}=d_{H}\left(v_{1}\right)+\sum_{w \in N_{H}\left(v_{1}\right)} x_{w} . \\
& \mu(G) x_{v_{1}}=d_{G}\left(v_{1}\right)+\sum_{w \in N_{G}\left(v_{1}\right)} x_{w} .
\end{aligned}
$$

Since $d_{H}\left(v_{1}\right) \geq d_{G}\left(v_{1}\right), \sum_{w \in N_{H}\left(v_{1}\right)} x_{w}>\sum_{w \in N_{G}\left(v_{1}\right)} x_{w}$, so we have $\mu(H) x_{v_{1}}>$ $\mu(G) x_{v_{1}}$. Since $x_{v_{1}}>0$, hence $\mu(H)>\mu(G)$, a contradiction.

Now, we consider the graph $G_{u v}$ obtained from the connected graph $G$ by subdividing the edge $u v$, that is, by replacing $u v$ with edges $u w, v w$, where $w$ is an additional vertex. We call the following two types of paths internal paths: (a) A sequence of vertices $v_{0}, v_{1}, \cdots, v_{k+1}(k \geq 2)$, where $v_{0}, v_{1}, \cdots, v_{k}$ are distinct, $v_{k+1}=v_{0}$ of degree at least $3, d_{v_{i}}=2$ for $i=1, \cdots, k$, and $v_{i-1}$ and $v_{i}(i=$ $1, \cdots, k+1)$ are adjacent. (b) A sequence of distinct vertices $v_{0}, v_{1}, \cdots, v_{k+1}(k \geq$ $0)$ such that $v_{i-1}$ and $v_{i}(i=1, \cdots, k+1)$ are adjacent, $d_{v_{0}} \geq 3, d_{v_{k+1}} \geq 3$ and $d_{v_{i}}=2$ whenever $1 \leq i \leq k$.

Lemma 2.3([8], [2]). Let $G$ be a connected graph and uv be some edge on the internal path of $G$ as we defined above. If we subdivide uv, that is, substitute it by $u w, w v$, with the new vertex $w$, and denote the new graph by $G_{u v}$, then $\mu\left(G_{u v}\right)<$ $\mu(G)$.

Lemma 2.4. Let $G$ be a connected graph. Suppose $v_{1}$ and $v_{2}$ are vertices each of degree at least three and $v_{1} v_{2}$ is an edge of $G$. Let $G^{\prime}$ be the connected graph obtained form $G$ by contracting $v_{1} v_{2}$ (i.e., deleting the edge and identifying $v_{1}$ and $\left.v_{2}\right)$. Then $\mu(G)<\mu\left(G^{\prime}\right)$.

Proof. The proof of the result is similar to Theorem 4.11 in [14] and we omit it.

Lemma 2.5([2]). Suppose $G$ is a nontrivial simple connected graph. Let u be a 
vertex of $G$. For nonnegative integers $k, l$, let $G(k, l)$ denote the graph obtained from $G$ by adding pendant paths of length $k$ and $l$ at $u$. If $k \geq l \geq 1$, then

$$
\mu(G(k, l))>\mu(G(k+1, l-1)) .
$$

Lemma 2.6. Let $G$ be a connected graph and $P$ be a pendant path in $G$. Suppose e is an edge in $P$ and $G^{\prime}$ is the graph obtained from $G$ by subdividing e, then we have $\mu(G)<\mu\left(G^{\prime}\right)$.

Proof. Since $G$ is a proper subgraph of $G^{\prime}$, we have $\mu(G)<\mu\left(G^{\prime}\right)$.

\section{Main results}

Suppose the vertices of the graphs $B(4,1,4), B(4,1,3), B(3,1,3)$ are labeled as in Fig. 1.

Let $B_{1}$ be the graph on $n$ vertices obtained from $B(4,1,4)$ by attaching $k$ paths of almost equal lengths (i.e., the lengths of these paths differ in size by at most one) at $u ; B_{2}$ be the graph obtained from $B(4,1,4)$ by attaching $k$ paths of almost equal lengths at $u_{4} ; B_{3}$ be the graph obtained from $B(4,1,4)$ by attaching $k$ paths of almost equal lengths at $u_{6}$.

Let $C_{1}$ be the graph on $n$ vertices obtained from $B(4,1,3)$ by attaching $k$ paths of almost equal lengths at $u ; C_{2}$ be the graph on $n$ vertices obtained from $B(4,1,3)$ by attaching $k$ paths of almost equal lengths at $u_{2} ; C_{3}$ be the graph obtained from $B(4,1,3)$ by attaching $k$ paths of almost equal lengths at $u_{5} ; C_{4}$ be the graph obtained from $B(4,1,3)$ by attaching $k$ paths of almost equal lengths at $u_{4}$.

Let $D_{1}$ be the graph on $n$ vertices obtained from $B(3,1,3)$ by attaching $k$ paths of almost equal lengths at $u ; D_{2}$ be the graph on $n$ vertices obtained from $B(3,1,3)$ by attaching $k$ paths of almost equal lengths at $u_{2}$.

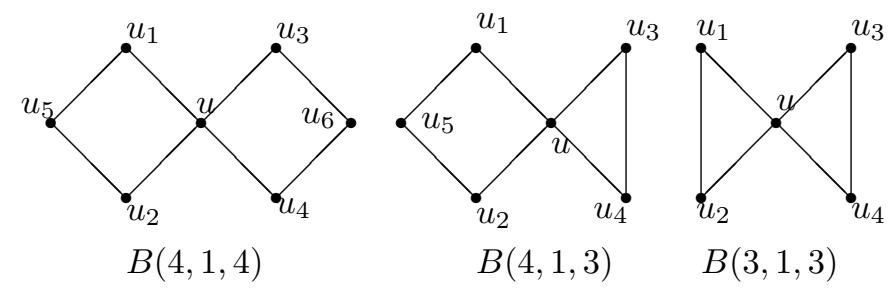

Fig.1

Theorem 3.1. Let $G$ be a bicyclic graph in $\mathcal{B}_{n}^{+}(k)$. Then $\mu(G) \leq \mu\left(D_{1}\right)$. The equality holds if and only if $G \cong D_{1}$.

Proof. Let $G$ be a bicyclic graph in $\mathcal{B}_{n}^{+}(k)$. Comparing the eigencomponents of the vertices on $B(p, l, q)$, by Lemma 2.2 , identifying the roots of the trees attached 
to $B(p, l, q)$, the signless Laplacian spectral radius increases. Next, by Lemmas 2.3 , 2.4, contracting the internal path and Lemma 2.5 to make all the pendant paths having almost equal lengths, the signless Laplacian spectral radius again increases. At last, subdividing the pendant paths several times if necessary to keep the order of graphs unchanged, by Lemma $2.6, \mu(G)$ increases.

So we conclude the following three cases hold.

(1). If $p \geq q \geq 4$ and $l \geq 1$, then $\mu(G) \leq \max \left\{\mu\left(B_{1}\right), \mu\left(B_{2}\right), \mu\left(B_{3}\right)\right\}$.

(2). If $p=4, q=3$ and $l \geq 1$, then $\mu(G) \leq \max \left\{\mu\left(C_{1}\right), \mu\left(C_{2}\right), \mu\left(C_{3}\right), \mu\left(C_{3}\right)\right\}$.

(3). If $p=q=3, l \geq 1$, then $\mu(G) \leq \max \left\{\mu\left(D_{1}\right), \mu\left(D_{2}\right)\right\}$.

For case (1), we claim that $\max \left\{\mu\left(B_{1}\right), \mu\left(B_{2}\right), \mu\left(B_{3}\right)\right\}=\mu\left(B_{1}\right)$.

In fact, for $B_{2}$, consider the eigencomponents corresponding to $u$ and $u_{4}$, say, $x_{u}$ and $x_{u_{4}}$. If $x_{u} \geq x_{u_{4}}$, by Lemma 2.1, removing the $k$ pendant paths to $u$, we have $\mu\left(B_{2}\right)<\mu\left(B_{1}\right)$. If $x_{u}<x_{u_{4}}$, by Lemma 2.1, deleting edges $u u_{1}, u u_{2}$ and adding edges $u_{4} u_{1}, u_{4} u_{2}$, we also have $\mu\left(B_{2}\right)<\mu\left(B_{1}\right)$. Similarly, for $B_{3}$, consider the eigencomponents corresponding to $u$ and $u_{6}$, we have $\mu\left(B_{3}\right)<\mu\left(B_{1}\right)$.

For case $(2)$, we claim that $\max \left\{\mu\left(C_{1}\right), \mu\left(C_{2}\right), \mu\left(C_{3}\right), \mu\left(C_{4}\right)\right\}=\mu\left(C_{1}\right)$.

In fact, for $C_{2}$, consider the eigencomponents corresponding to $u$ and $u_{2}$, say, $x_{u}$ and $x_{u_{2}}$. If $x_{u} \geq x_{u_{2}}$, by Lemma 2.1, removing the $k$ pendant paths to $u$, we have $\mu\left(C_{2}\right)<\mu\left(C_{1}\right)$. If $x_{u}<x_{u_{2}}$, by Lemma 2.1, deleting edges $u u_{3}, u u_{4}$ and adding edges $u_{2} u_{3}, u_{2} u_{4}$, we also have $\mu\left(C_{2}\right)<\mu\left(C_{1}\right)$. Similarly, for $C_{3}$, consider the eigencomponents corresponding to $u$ and $u_{6}$, we have $\mu\left(C_{3}\right)<\mu\left(C_{1}\right)$; for $C_{4}$, consider the eigencomponents corresponding to $u$ and $u_{4}$, we have $\mu\left(C_{4}\right)<\mu\left(C_{1}\right)$.

For case (3), we claim that $\max \left\{\mu\left(D_{1}\right), \mu\left(D_{2}\right)\right\}=\mu\left(D_{1}\right)$.

This is similar to the above two cases.

At last, we claim that $\max \left\{\mu\left(B_{1}\right), \mu\left(C_{1}\right), \mu\left(D_{1}\right)\right\}=\mu\left(D_{1}\right)$.

In fact, for $C_{1}$, by Lemma 2.3 , contracting edge $u_{1} u_{5}$ and by Lemma 2.6, subdividing the pendant edge one time, by Lemma 2.5, we get the graph $D_{1}$ and $\left.\mu\left(C_{1}\right)\right\}<\mu\left(D_{1}\right)$.

For $B_{1}$, contracting edge $u_{1} u_{5}, u_{3} u_{6}$ and by Lemma 2.6 , subdividing the pendant edge one time, by Lemma 2.5, we get the graph $D_{1}$ and $\left.\mu\left(B_{1}\right)\right\}<\mu\left(D_{1}\right)$.

Suppose the vertices of the graphs $P(3,1,3), P(3,1,2), P(2,1,2)$ are labeled as in Fig. 2.

Let $E_{1}$ be the graph obtained from $P(2,1,2)$ by attaching $k$ paths of almost equal lengths at $u_{3} ; E_{2}$ be the graph obtained from $P(2,1,2)$ by attaching $k$ paths of almost equal lengths at $u_{4}$.

Let $F_{1}$ be the graph on $n$ vertices obtained from $P(3,1,2)$ by attaching $k$ paths of almost equal lengths at $u_{3} ; F_{2}$ be the graph on $n$ vertices obtained from $P(3,1,2)$ by attaching $k$ paths of almost equal lengths at $u_{4} ; F_{3}$ be the graph obtained from $P(3,1,2)$ by attaching $k$ paths of almost equal lengths at $u_{5}$.

Let $G_{1}$ be the graph on $n$ vertices obtained from $P(3,1,3)$ by attaching $k$ paths of almost equal lengths at $u_{4} ; G_{2}$ be the graph on $n$ vertices obtained from $P(3,1,3)$ by attaching $k$ paths of almost equal lengths at $u_{3}$. 


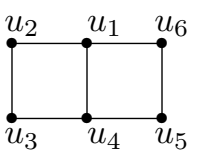

$P(3,1,3)$

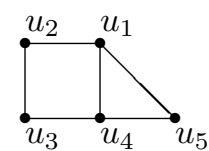

$P(3,1,2)$

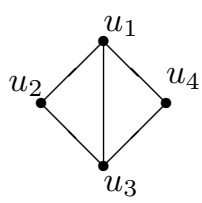

$P(2,1,2)$

Fig.2

Theorem 3.2. Let $G$ be a bicyclic graph in $\mathcal{B}_{n}^{++}(k)$. Then $\mu(G) \leq \mu\left(E_{1}\right)$. The equality holds if and only if $G \cong E_{1}$.

Proof. Similar as in Theorem 3.1, we conclude that the following three cases holds.

(1). If $p=l=2$ and $q=1$, then $\mu(G) \leq \max \left\{\mu\left(E_{1}\right), \mu\left(E_{2}\right)\right\}$.

(2). If $p=3, l=2$ and $q=1$ or 2 , then $\mu(G) \leq \max \left\{\mu\left(F_{1}\right), \mu\left(F_{2}\right), \mu\left(F_{3}\right)\right\}$.

(3). If $p \geq l \geq 3, q \geq 1$, then $\mu(G) \leq \max \left\{\mu\left(G_{1}\right), \mu\left(G_{2}\right)\right\}$.

For case (1), we claim that $\max \left\{\mu\left(E_{1}\right), \mu\left(E_{2}\right)\right\}=\mu\left(E_{1}\right)$

In fact, in $E_{2}$, just consider the eigencomponents of $u_{3}$ and $u_{4}$, by Lemma 2.1, we can get the claim.

For case (2), we claim that $\max \left\{\mu\left(F_{1}\right), \mu\left(F_{2}\right), \mu\left(F_{3}\right)\right\}=\mu\left(F_{2}\right)$.

In fact, in $F_{1}$, just consider the eigencomponents of $u_{3}$ and $u_{1}$, by Lemma 2.1, we get $\mu\left(F_{1}\right) \leq \mu\left(F_{2}\right)$; in $F_{3}$, consider the eigencomponents of $u_{1}$ and $u_{5}$, by Lemma 2.1 , we get $\mu\left(F_{3}\right) \leq \mu\left(F_{2}\right)$, as claimed.

For case (3), we claim that $\max \left\{\mu\left(G_{1}\right), \mu\left(G_{2}\right)\right\}=\mu\left(G_{1}\right)$.

In fact, in $G_{2}$, just consider the eigencomponents of $u_{3}$ and $u_{1}$, by Lemma 2.1, we can get the claim.

At last, we claim that $\max \left\{\mu\left(E_{1}\right), \mu\left(F_{2}\right), \mu\left(G_{1}\right)\right\}=\mu\left(E_{1}\right)$.

In fact, for $F_{2}$, by Lemma 2.3 , contracting edge $u_{2} u_{3}$ and by Lemma 2.6, subdividing the pendant edge one time, by Lemma 2.5 , we get the graph $E_{1}$ and $\left.\mu\left(F_{2}\right)\right\}<\mu\left(E_{1}\right)$.

For $G_{1}$, contracting edge $u_{2} u_{3}, u_{5} u_{6}$ and by Lemma 2.6 , subdividing the pendant edge one time, by Lemma 2.5, we get the graph $E_{1}$ and $\left.\mu\left(G_{1}\right)\right\}<\mu\left(E_{1}\right)$.

Lemma 3.3([4]). Let $G$ be a graph on $n$ vertices with at least one edge and the maximum degree of $G$ be $\Delta$. Then $\mu(G) \geq \Delta+1$. The equality holds if and only if $G$ is a star.

Lemma 3.4([9]). For a connected graph $G$, we have $\mu(G) \leq \max \left\{d_{u}+m_{u}: u \in\right.$ $V(G)\}$, where $m_{u}$ satisfies $d_{u} m_{u}=\sum_{v u \in E(G)} d_{v}$. The equality holds if and only if $G$ is regular or semiregular bipartite.

Theorem 3.5. Let $G$ be a bicyclic graph in $\mathcal{B}_{n}(k)$. Then $\mu(G) \leq \mu\left(D_{1}\right)$, the equality holds if and only if $G=D_{1}$.

Proof. $\quad$ By Theorems 3.1, 3.2, we have $\mu(G) \leq \max \left\{\mu\left(D_{1}\right), \mu\left(E_{1}\right)\right\}$. For $D_{1}$, by Lemma 3.3, we have $\mu\left(D_{1}\right) \geq k+5$. By Lemma $3.4, \mu\left(E_{1}\right)<k+5$. This implies 
the result.

Acknowledgment. The author is grateful to the referee for his or her valuable suggestions which lead to an improvement of this paper.

\section{References}

[1] J. A. Bondy, U.S.R. Murty, Graph Theory with Applications, Macmillan Press, New York, 1976

[2] D. Cvetković, S.K. Simic, Towards a spectral theory of graphs based on the signless Laplacian, Publ. Inst. Math. (Beograde), 85(99),19-33.

[3] D. Cvetković, Signless Laplacians and line graphs, Bull. Acad. Serbe Sci.Ars. Cl. Sci. Math. Nat. Sci. Math., 131(30)(2005), 85-92.

[4] D. Cvetković, P.Rowlinson, S.K.Simić, Eigenvalue bounds for the signless Laplacian, Publ. Inst. Math. (Beograd), 81(95)(2007), 11-27.

[5] D. Cvetković, P. Rowlinson, S. K. Simić, Signless Laplacians of finite graphs, Linear Algebra Appl., 423(2007), 155-171.

[6] M. Desai, V. Rao, A characterizaion of the smallest eigenvalue of a graph, J. Graph Theory, 18(1994), 181-194.

[7] Y. Fan, B.S. Tam, J. Zhou, Maximizing spectral radius of unoriented Laplacian matrix over bicyclic graphs of a given order, Linear Multilinear Algebra, 56(2008), 381-397.

[8] L. Feng, Q. Li, X. Zhang, Minimizing the Laplacian spectral radius of trees with given matching number, Linear and Multilinear Algebra, 55(2007), 199-207.

[9] L. Feng, Q. Li, X. Zhang, Some sharp upper bounds on the spectral radius of graphs, Taiwanese J. Math., 11(2007), 989-997.

[10] L. Feng, G. Yu, No starlike trees are Laplacian cospectral, Univ. Beograd. Publ. Elektrotehn. Fak. Ser. Mat., 18(2007), 46-51.

[11] L. Feng, G. Yu, The signless Laplacian spectral radius of graphs with given diameter, Utilitas Mathematica, to appear.

[12] J. W. Grossman, D.M. Kulkarni, I.Schochetman, Algebraic graph theory without orientation, Linear Algebra Appl. 212/213(1994), 289-307.

[13] J. Guo, The Laplacian spectral radius of a graph under perturbation, Comput. Math. Appl., 54(2007), 709-720.

[14] R. Grone, R. Merris, V. S. Sunder. The Laplacian spectrum of a graph, SIAM J. Matrix Anal. Appl., 11(1990), 218-238.

[15] R. Grone, R. Merris, The Laplacian spectrum of a graph II, SIAM J. Discrete Math., 7(1994), 221-229.

[16] S. G. Guo, The spectral radius of unicyclic and bicyclic graphs with $n$ vertices and $k$ pendant vertices, Linear Algebra Appl., 408(2005), 78-85. 
[17] S.G. Guo, The largest eigenvalues of the Laplacian matrices of unicyclic graphs, Appl. Math. J. Chinese Univ. Ser. A, 16(2001), no. 2, 131-135. (in Chinese) MR2002b:05096.

[18] C.X. He, J.Y. Shao, J.L. He, On the Laplacian spectral radii of bicyclic graphs, Discrete Math., 308(2008), 5981-5995.

[19] Y. Hong, X.-D. Zhang, Sharp upper and lower bounds for largest eigenvalue of the Laplacian matrices of trees, Discrete Math., 296(2005), 187-197.

[20] R. Merris, Laplacian matrices of graphs: a survey, Linear Algebra Appl., 197/198(1994), 143-176. 